\title{
Quantités ingérées et croissance de jeunes bisonnes d'élevage : effet de la saison
}

\author{
J Agabriel ${ }^{1}$, J Bony ${ }^{2}$, M Petit ${ }^{1}$ \\ ${ }^{1}$ Laboratoire adaptation des herbivores aux milieux, Inra, 63122 Saint-Genès-Champanelle; \\ 2 Inra, domaine expérimental des Razats, 63830 Laqueuille, France
}

(Reçu le 3 avril 1995 ; accepté le 20 janvier 1996)

\begin{abstract}
Résumé - Pour mieux connaître le niveau d'ingestion et la croissance de jeunes bisonnes d'élevage (Bison bison) et leur sensibilité à la longueur du jour, $2 \times$ quatre femelles âgées de 12 à 16 mois ont été placées en stabulation libre sur aire paillée dans deux cases d'environ $30 \mathrm{~m}^{2}$ chacune : lot L1, -lourdes- $P V=249 \mathrm{~kg}$; lot $L 2$, -légères- $P V=187 \mathrm{~kg}$. Le lot $L 1$ a reçu un foin de première coupe $(\mathrm{DMO}=0,57)$ et le lot $\mathrm{L} 2$ un foin de deuxième coupe $(\mathrm{DMO}=0,685)$ de la même prairie. Les quantités ingérées (Q1) ont été mesurées quotidiennement pendant 34 semaines de septembre à mai. Les animaux ont été pesés chaque mois. Les QI moyennes ont été respectivement de $5,6 \pm 0,5$ et $5,2 \pm 0,3$ $\mathrm{kg} \mathrm{MS} /$ jour, et les GMQ correspondants de $202 \pm 45 \mathrm{~g} /$ jour (lot L1) et de $374 \pm 107 \mathrm{~g} / \mathrm{jour}$ (lot L2). Le niveau d'ingestion (par rapport au poids vif) est supérieur de 20 à $25 \%$ aux prévisions des modèles bovins (Inra, 1988) pour des "génisses rustiques" de même âge et qui recevraient ces mêmes foins. Mais les QI et GMQ ont fortement évolué au cours de l'expérience et en fonction de la saison. Trois périodes ont été distinguées : $P 1$, jusqu'au 2 décembre, $P 2$ jusqu'au 1er février et $P 3$ jusqu'au 15 mai. Les $\mathrm{Q}$ l baissent entre $\mathrm{P} 1$ et $\mathrm{P} 2$ de $-0,8$ (lot L1) et de $-0,9$ (lol L2) $\mathrm{kg} \mathrm{MS/jour,} \mathrm{puis} \mathrm{augmentent} \mathrm{entre}$ $\mathrm{P} 2$ et $\mathrm{P} 3$ de $+0,8$ et $+0,1 \mathrm{~kg} \mathrm{MS} / \mathrm{jour}$. Les $\mathrm{GMQ}$ ont été maximum en $\mathrm{P} 1$ (437 et $595 \mathrm{~g} / \mathrm{jour}$ ), minimum en P2 (-157 et $144 \mathrm{~g} / \mathrm{jour}$ ) et de nouveau plus élevés en P3 (234 et $336 \mathrm{~g} / \mathrm{jour}$ ). Proportionnellement, la variation saisonnière de la croissance semble plus élevée que celle du niveau d'ingestion. En P1 et P3 la relation entre l'énergie ingérée et la croissance semble identique à celle retenue pour des génisses de $250 \mathrm{~kg}$, ce qui n'est plus vrai en P2 : cela pose le problème du devenir de l'énergie ingérée en période de jours courts.
\end{abstract}

\section{capacité d'ingestion / croissance / bison}

Summary - Seasonal variations of intake and growth of young female bison. To describe the intake capacity and the growth potential of young female bison (Bison bison), $2 \times 4$ bison heifers from 12 to 16 months old $(L 1$, tall, live weight $249 \mathrm{~kg}$; and $L 2$, small, live weight $187 \mathrm{~kg})$ were fed indoors on a hay diet given ad libitum during 34 weeks. Dry matter intake (DMI) was measured daily from September to May. The L1 group received a natural grassland hay ( $F 1$ : first cut, organic matter digestibility [OMD] $=0.570$ ) and the $L 2$ group received the second cut, from the same field ( $F 2$ : OMD $=0.685)$. These hays were also given to Salers heifers in order to determine their feeding values. Bisons were weighed 
every month. DMI were, respectively, $5.6 \pm 0.5$ and $5.2 \pm 0.3 \mathrm{~kg} /$ day and average daily gains (ADG) were $202 \pm 45 \mathrm{~g} /$ day (group L1) and $374 \pm 107 \mathrm{~g} /$ day (group L2). The intake capacity per $\mathrm{kg}$ of live weight is 20 to $25 \%$ higher than those calculated by bovine model (INRA, 1988) for hardy breed heifers at the same age receiving these hays. DMI and ADG varied widely during the trial with the season (fig 1). Three periods were described: P1 from the beginning to December, $P 2$ to February and P3 up to 15 May. DMI decreased from $P 1$ to $P 2-0.8 \mathrm{~kg} /$ day $(L 1)$ and $-0.9 \mathrm{~kg} /$ day $(L 2)$, then increased between $P 2$ and $P 3$ +0.8 and $+0.1 \mathrm{~kg} \mathrm{DM} /$ day (table l). ADG were maximum during $P 1$ (437 and $595 \mathrm{~g} /$ day), minimum during $P 2$ (-157 and $144 \mathrm{~g} /$ day) and high again during $P 3$ (234 and $336 \mathrm{~g} /$ day). Seasonal variations of growth level seem to be proportionally more important than seasonal variations of appetite. During $P 1$ and $P 3, A D G$ were linearly related to energy supply, in similar manner for the young bison female than for a $250 \mathrm{~kg}$ heifer, but this was not true in P2 (fig 2). Further investigations are needed in order to describe the diet energy utilisation during winter by the bisons.

intake capacity / growth / bison

\section{INTRODUCTION}

La capacité d'ingestion des bisons d'Amérique (Bison bison) est encore mal connue. Elle semble plus faible que celle des bovins puisque dans des comparaisons entre espèces, les quantités ingérées de foins par les bisons rapportées au poids vif sont inférieures $(-11 \%$ Towne et al, 1988$)$, ou au mieux égales à celles de bovins Hereford (Richmond et al, 1977) pour des foins de diverses qualités (Towne et al, 1989). Cette différence s'expliquerait en partie par la taille du rumen proportionnellement plus faible chez le bison. La capacité d'ingestion des bisons varierait aussi suivant les saisons : lorsque des bisons et des bovins reçoivent des fourrages identiques en hiver ou en été, l'écart d'ingestion entre les deux espèces semble s'accroître durant l'hiver (Hawley et al, 1981b).

Dans des conditions d'élevage extensif américain, le poids des femelles bisonnes à 1 an est d'environ $200 \mathrm{~kg}$ ce qui ne représenterait que 40 à $45 \%$ du poids adulte atteint au-delà de 6 ans (Jenning and Hebbring, 1983). Les bisons ont donc sans doute un potentiel de croissance modéré en grande partie lié à leur faible format adulte, et peut être à leur faible précocité.
Mais les faibles niveaux de croissance observés peuvent aussi résulter des effets cumulés des ressources alimentaires restreintes, de la baisse du niveau d'ingestion en hiver et de l'augmentation des besoins liée aux déplacements ou à la lutte contre le froid. Enfin comme chez les cervidés (Thériez, 1989), la longueur du jour pourrait avoir un effet direct sur la capacité de croissance. Le but de cette expérience est de quantifier l'effet de la saison sur le niveau d'ingestion et sur la vitesse de croissance de jeunes femelles bisonnes, placées en stabulation libre dans des conditions d'alimentation non restrictive.

\section{MATÉRIEL ET MÉTHODES}

Huit génisses bisonnes (Bison bison) âgées de 12 à 16 mois environ ont été placées le $20 \mathrm{sep}$ tembre 1993 en stabulation libre sur aire paillée dans deux cases d'environ $30 \mathrm{~m}^{2}$ chacune, à l'intérieur d'un hangar fermé, naturellement ventilé, et bien éclairé par la lumière du jour. Pour limiter les effets de hiérarchie, les plus lourdes (lot $\mathrm{L} 1, n=4$, poids vif initial moyen (PV) $=249 \mathrm{~kg}$ ) et les plus légères d'entre elles (lot $L 2, n=4$, $P V=187 \mathrm{~kg}$ ) ont été séparées. Ces génisses étaient arrivées sur l'exploitation 7 mois plus tôt et avaient pâturé ensemble de mai à septembre. Leur croissance moyenne au pâturage (138 jours) avait été respectivement de 260 et $420 \mathrm{~g} / \mathrm{jour}$. 
Après une semaine d'adaptation, elles ont reçu pendant 34 semaines, du 27 septembre 1993 au 15 mai 1994, du foin de prairie permanente de montagne distribué à volonté et $0,1 \mathrm{~kg}$ de complément minéral vitaminé. Le lot $L 1$ a reçu un foin de première coupe ( $F 1$, récolté le 26 juin, $M A T=99 \mathrm{~g}$ et $C B=379 \mathrm{~g} / \mathrm{kg} \mathrm{MS}$ ), et le lot L2 le foin de deuxième coupe de la même parcelle ( $F 2$, récolté le 20 août, $M A T=146 \mathrm{~g}, \mathrm{CB}=296 \mathrm{~g} / \mathrm{kgMS}$ ). La digestibilité de la MO des foins (DMO), mesurée sur un lot de six moutons, a été respectivement de 0,57 et 0,685 . Pour préciser leurs valeurs alimentaires, ces deux foins ont été distribués parallèlement pendant 15 semaines à deux lots de 14 génisses bovines Salers âgées de 7 à 11 mois. A partir des quantités ingérées ( 3,6 et $3,7 \mathrm{~kg}$ $\mathrm{MS} /$ jour de foin $\mathrm{F} 1$ et $F 2$ ) et des croissances ( 630 et $840 \mathrm{~g} / \mathrm{jour}$ ) de ces génisses, nous avons retenu respectivement pour $F 1$ et $F 2$ les valeurs énergétiques de 0,66 et 0,74 UFL $/ \mathrm{kg}$ MS $(7,8$ et $8,8 \mathrm{MJ}$ EM/kg MS) et les valeurs d'encombrement (Dulphy et al, 1987) de 1,16 et $1,10 \mathrm{UEB} / \mathrm{kg}$ MS, soit des écarts entre foins inférieurs à ceux que les DMO mesurées sur les moutons laissaient prévoir.

La ration journalière était distribuée à $9 \mathrm{~h}$ et $16 \mathrm{~h}$. Les refus (qui représentaient environ $10 \%$ de la quantité offerte) ont été mesurés 5 jours par semaine. Les quantités ingérées ont été calculées pour chaque lot et chaque semaine. Les animaux ont été pesés une fois par mois.

Au vu de leurs évolutions, les données concernant l'ingestion et la croissance ont été regroupées a posteriori en 3 périodes : $P 1$ regroupant les 10 premières semaines jusqu'au 2 décembre, $P 2$ les 9 semaines suivantes jusqu'au 1 er février, et $\mathrm{P} 3$ les 15 dernières semaines. Les croissances individuelles ont été analysées par analyse de variance (Sas GLM) en intégrant dans le modèle les effets fixes indissociables de la case et du foin ( $F 1$ F2), de la période ( $P 1$ à $P 3$ ) et la croissance au cours de la période de pâturage précédent comme covariable, pour s'affranchir des effets de croissance compensatrice.

\section{RÉSULTATS}

Les quantités moyennes ingérées (QI) par les lots $L 1$ et $L 2$ sur l'ensemble de la période de mesure ont été respectivement de 5,6 et $5,2 \mathrm{~kg} \mathrm{MS} / \mathrm{jour}$, soit $2,00 \pm 0,14$ (L1) et $2,25 \pm 0,15$ (L2) $\mathrm{kg} \mathrm{MS} / 100 \mathrm{~kg}$ de poids vif. Elles ont fortement évolué au cours de l'expérience. D'un maximum voisin de $6,0 \mathrm{~kg}$ MS pour les deux lots au cours des cinq premières semaines elles sont passées par un minimum de $4,7 \mathrm{~kg}$ au cours du mois de janvier (tableau I et fig 1). Les QI ont ainsi baissé de $-0,8$ (lot L1) et de $-0,9$ (lot L2) kg MS/jour entre P1 et P2. Quoique plus légères, les bisonnes $L 2$ ont consommé en $\mathrm{P} 1$ et $\mathrm{P} 2$ autant que les bisonnes L1. Les QI se sont ensuite accrues de février à mai (entre P2 et P3), plus rapidement et de façon plus importante pour le lot $L 1 \quad(+0,8$ $\mathrm{kg} \mathrm{MS/jour)} \mathrm{que} \mathrm{pour} \mathrm{le} \mathrm{lot} \mathrm{L2}(+0,1 \mathrm{~kg}$ $\mathrm{MS} / \mathrm{jour}$ ). Exprimés en $\mathrm{kg}$ de MS par 100 $\mathrm{kg}$ de poids vif, les niveaux d'ingestion des lots $L 1$ et $L 2$ suivent la même évolution que les valeurs brutes.

Tableau I. Évolution des performances des bisonnes selon la période.

\begin{tabular}{|c|c|c|c|c|c|c|}
\hline & $\cdots--$ & - &.- & - & - & - \\
\hline & & Lot $L 1$ & & & Lot $L 2$ & \\
\hline Période & $P 1$ & $P 2$ & P3 & $P 1$ & $P 2$ & P3 \\
\hline - & & & & & & $-\ldots$ \\
\hline Poids moyen (kg) & $264 \pm 18$ & $260 \pm 19$ & $281 \pm 20$ & $208 \pm 22$ & $233 \pm 25$ & $251 \pm 30$ \\
\hline GMQ (g/jour) 4 & $437^{a} \pm 68$ & $-157^{b} \pm 67$ & $234^{a} \pm 96$ & $595^{a} \pm 161$ & $144^{b} \pm 49$ & $336^{a} \pm 124$ \\
\hline MSI (kg/jour) & 5,7 & 4,9 & 5,7 & 5,8 & 4,9 & 5,0 \\
\hline
\end{tabular}

Des lettres différentes correspondent à des différences significatives entres périodes $p<0,05 ; \mathrm{P} 1: 27$ sept-2 déc ; $P 2: 3$ déc-1er fév; P3: 2 fév-15 mai. 
Sur la durée de l'essai, les croissances moyennes ont été de $202 \pm 45 \mathrm{~g} / \mathrm{jour}$ (lot L1) et de $374 \pm 107 \mathrm{~g} /$ jour (lot L2). Comme les quantités ingérées, ces croissances n'ont pas été régulières (fig 1 ) et semblent affectées par la durée du jour. Maximales en P1 (respectivement : 437 et $595 \mathrm{~g} /$ jour), elles ont été significativement $(p<0,05)$ plus faibles en P2 (lot L2 : $144 \mathrm{~g} /$ jour), voire négatives (lot L1 : $-157 \mathrm{~g} / \mathrm{jour}$ ). En P3 les croissances sont redevenues plus élevées sans toutefois atteindre le niveau de la période 1 (234 et $336 \mathrm{~g} / \mathrm{jour}$ pour les lots L1 et L2).

La variabilité des croissances est élevée entre individus: $\mathrm{cV}=22,3 \%$ pour le lot $\mathrm{L} 1$ et $\mathrm{cv}=28,6 \%$ pour le lot $\mathrm{L} 2$. Elle peut en partie résulter de relations exacerbées de dominance-soumission dans chaque lot,

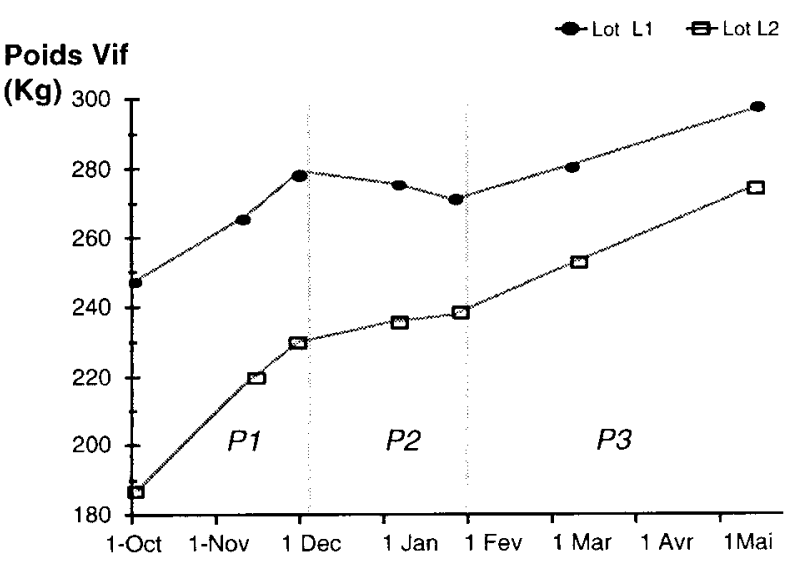

M.S.I

$(\mathrm{kg} / \mathrm{j})$

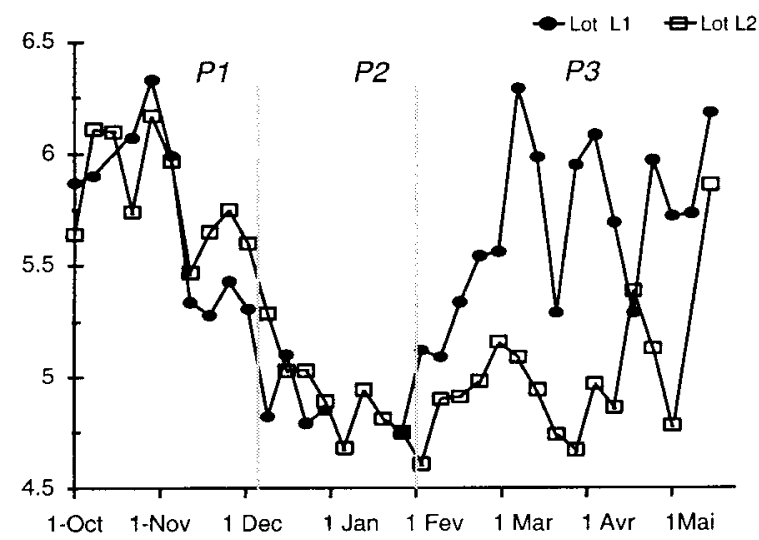

Durée du jour (h)

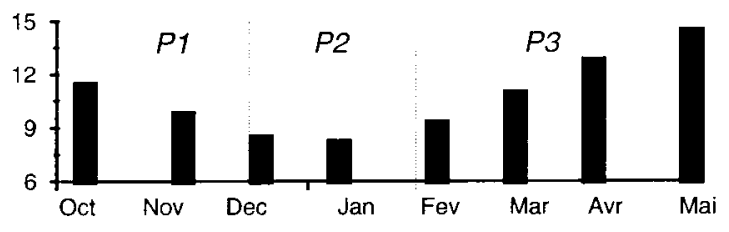

Fig 1. Évolution des poids vifs $(\mathrm{kg})$ et des quantités de foin ingérées $(\mathrm{kg}$ MS/jour) des deux lots de bisonnes selon la date et la durée du jour. P1 : 27 sept-2 déc; $\mathrm{P} 2$ : 3 déc-1er fév ; P3: 2 fév-15 mai. 
quelques bisonnes ayant semblé particulièrement brimées (coups répétés) par d'autres. La croissance au cours de la saison de pâturage précédente influe d'ailleurs positivement $(p>0,01)$ sur les croissances hivernales, il n'y a pas de compensation : ce sont toujours les mêmes bisonnes qui ont la meilleure croissance.

\section{DISCUSSION}

\section{Quantités ingérées}

Rapportées au poids vif, les quantités moyennes de foin ingéré $(2,00$ et $2,25 \mathrm{~kg}$ MS/100 kg PV pour L1 et L2) ont été logiquement plus élevées avec le foin $\mathrm{F} 2$ qui était de meilleure qualité. Ce niveau d'ingestion correspond aux observations antérieures faites sur des bisons européens (Bison bonasus) par Gebczynska et al $(1972,1974)$ et sur des bisons d'Amérique par Towne et al (1989), Hawley et al (1981b). Pour ces derniers, les QI de bisons pesant 300 à $375 \mathrm{~kg}$ variaient de 1,60 à $2,37 \mathrm{~kg} \mathrm{MS} / 100 \mathrm{~kg} \mathrm{PV}$, avec des foins dont la teneur en MAT allait de 8 à $22 \%$.

À l'inverse de Hawley et al (1981a), le niveau moyen d'ingestion des bisonnes au cours de l'expérience a été supérieur à celui estimé pour des génisses de l'espèce bovine. En utilisant les valeurs d'encombrement des foins $(1,16$ et $1,10 \mathrm{UEB} / \mathrm{kg}$ $\mathrm{MS})$, la capacité d'ingestion (CI) des bisonnes serait de 2,32 (lot L1) et 2,48 (lot L2) UEB/100 kg PV. Cette Cl est supérieure à celle de génisses Salers ou Charolaise d'âges comparables ( 1,9 à 2,0 UEB/100 kg $\mathrm{PV}$ ), et voisine de celle de génisses Holstein. Cela reste vrai en période $\mathrm{P} 2$ où les $\mathrm{Q}$ étaient les plus faibles.

Les quantités ingérées ont varié dans le même sens que la durée du jour (fig 1). Elles ont ainsi baissé de plus de $20 \%$ entre octobre et janvier. Ces variations de QI sont beaucoup plus importantes que celles observées sur des bovins: +6 à $+8 \%$ pour des génisses Holstein de $250 \mathrm{~kg}$ quand la durée d'éclairement passe de 8 à 16 heures par 24 heures (Peters et al, 1980 ; Petitclerc et al, 1983). En revanche des variations comparables ( $+25 \%$ de février à août) ont été citées pour des moutons de race Texel (Michalet-Doreau et Gatel, 1988), qui restent bien inférieures aux observations sur les ovins Soay des Hébrides (Kay, 1985) ou sur les cervidés (environ $+50 \%$ entre l'hiver et l'été, Adam, 1987 ; Thériez, 1988).

Dans la présente expérience, les chutes de Q1 entre P1 et P2 ont pu être exacerbées par une surconsommation temporaire lors des 4 ou 5 premières semaines qui suivent la rentrée à l'étable et qui s'observe parfois chez les bovins (Coulon et al, 1986) après une saison de pâturage médiocre (le GMQ des bisonnes n'a été que de $340 \mathrm{~g} /$ jour au cours de l'été précédent). Cette surconsommation temporaire pourrait également provenir d'un comportement particulier des bisonnes stressées par l'espace restreint des cases.

Par la suite, entre P2 et P3, l'ingestion a plus augmenté $(+15 \%)$ pour les animaux L1 (foin moyen/grand format) que pour les animaux L2 (bon foin/ petit format), $+2 \%$. Les bisonnes L2 auraient atteint leur potentiel de croissance maximum (environ 350 $\mathrm{g} / \mathrm{jour}$ ), alors que les bisonnes L1 (230 $\mathrm{g} /$ jour) chercheraient à l'atteindre par une ingestion plus importante du foin $\mathrm{F} 1$ de moins bonne qualité .

\section{Croissance et utilisation de l'énergie}

Les croissances moyennes des lots $\mathrm{L} 1$ et L2 (200 à $375 \mathrm{~g} /$ jour) semblent faibles au regard des quantités ingérés. À même âge moyen (18 mois environ) des génisses bovines pesant $330 \mathrm{~kg}(60 \%$ d'un poids adulte de $550 \mathrm{~kg}$ ), et qui auraient reçu les deux mêmes foins expérimentaux, auraient 
réalisé des gains de $200 \mathrm{~g} / \mathrm{jour}(\mathrm{F} 1)$ et de 540 g/jour (F2) (Inra, 1988), mais en ingérant presque $20 \%$ de moins que les bisonnes par rapport au PV. Du fait d'un format adulte limité, et peut-être d'un développement lent, le potentiel de croissance des bisonnes (poids moyens à âges types) est vraisemblablement faible. L'âge moyen à l'abattage des mâles entiers sur les marchés américains (trois ans, d'après Benoteau, 1993) et leur faible poids de carcasse (250-300 kg pour un rendements de $56 \%$ ) en sont une illustration. Mais l'origine mal connue des animaux utilisés ici, et/ou une mauvaise adaptation à leur nouveau milieu, pourraient aussi être mises en cause.

La vitesse de croissance et la durée du jour varient dans le même sens (fig 1) ; sans que l'on puisse déterminer s'il s'agit d'un effet direct, ou si la baisse des QI en est la conséquence ou la cause. Si, pour chaque période, la quantité d'énergie ingérée par $\mathrm{kg} / \mathrm{P}^{0,75}$ est rapportée à la croissance journalière (fig 2), trois points ( $L 1$ en $P 1$, L1 et $L 2$ en $\mathrm{P} 3$ ) se situent à peu près sur la droite correspondant aux performances d'une génisse tardive ou rustique de $250 \mathrm{~kg}$ (Inra, 1988) : une croissance nulle des bisonnes serait alors obtenue avec un niveau d'apport énergétique de 0,051 UFL / $\mathrm{kgP}^{0,75}$. En P2, en revanche, les bisonnes ont particulièrement mal transformé l'énergie ingérée car leurs croissances sont inférieures de 150 à $200 \mathrm{~g}$ à celles espérées.

L'utilisation digestive du fourrage serait donc mauvaise et/ou l'utilisation de l'énergie digestible moins efficace. Cette baisse d'efficacité serait accompagnée d'une augmentation de la chaleur produite, bien que la réduction de la durée du jour soit par ailleurs connue pour diminuer le métabolisme basal, par exemple chez les cervidés et les ovins (Kay, 1985). Enfin, par rapport à l'énergie ingérée, les croissances du lot L2 ont également été faibles en période $\mathrm{P} 1$, et pourraient traduire une augmentation des besoins d'entretien liée au stress provoqué par le confinement.

\section{CONCLUSION}

Dans cet essai, les quantités ingérées de foin par $\mathrm{kg}$ de poids chez les jeunes bisonnes semblent en moyenne plutôt plus

\section{Énergie ingérée}

UFL ${ }^{*} 10^{-3} / \mathrm{kg}^{0.75}$

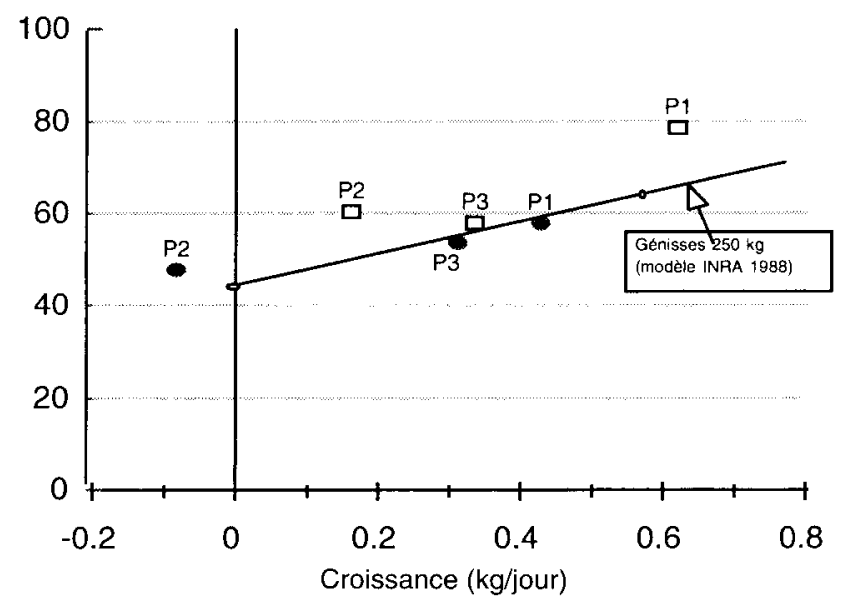

Fig 2. Énergie ingérée et croissance. 
élevées que les quantités ingérées par des génisses bovines rustiques qui auraient leur âge. L'énergie ingérée est, en revanche, mal valorisée par les bisonnes et leur potentiel de croissance semble faible.

Les bisonnes sont sensibles à la longueur du jour. Les quantités ingérées et la croissance diminuent en période de jours courts, mais toutefois dans une moindre proportion que chez les jeunes biches. La baisse de croissance journalière pendant l'hiver est proportionnellement plus forte que la baisse des quantités ingérées. Ces résultats n'avaient pas encore été mis en évidence, mais ils restent à confirmer sur des effectifs d'animaux plus conséquents.

Les niveaux d'ingestion et les croissances respectives des bisons et des bovins devront faire l'objet de comparaisons supplémentaires, pour mieux quantifier les écarts saisonniers entre les deux espèces, et mieux cerner le devenir de l'énergie ingérée par les bisons pendant la période hivernale.

\section{REMERCIEMENTS}

Cette étude a été réalisée grâce à l'appui financier du Fidar IR Massif central. Les auteurs tiennent à remercier particulièrement toute l'équipe technique de la ferme expérimentale de Laqueuille pour la contention et la manipulation des bisons.

\section{RÉFÉRENCES}

Adam CL (1987) Deer production. In: Consequences of milk quotas and alternative animal enterprises (JF O'Grady, ed), CEC seminar, Dublin 9-10 October 1986

Benoteau G (1993). La viande de bison américain d'élevage : étude d'opportunité d'exportation vers la CEE. Application au marché français et belge. Mémoire ESA, Angers

Coulon JB, D'hour P, Garel JP, Petit M (1986) Performance des vaches laitières autour de la rentrée à l'étable à l'automne : influence du type de ration de base et de la quantité de concentré offerte. Ann Zootech $35,37-48$
Dulphy JP, Faverdin P, Micol D, Bocquier F (1987) Révision du système des unités d'encombrement (UE) Bull Tech CRZV Theix 70, 35-48

Gebczynska Z, Krasinska M (1972). Food preferences and requirements of the European bison. Acta Theriologica 17, 105-117

Gebczynska Z, Kowalczyk J, Krasinska M, Ziolecka A (1974) A comparison of the digestibility of nutrients by European bison and cattle. Acta Theriologica 19, 283-289

Hawley AWL, Peden DG, Reynolds HW, Stricklin WR (1981a) Bison and cattle digestion of forages from the Slave River Lowlands, Northwest territories. Canada J Range Manage 34, 126-130

Hawley AWL, Peden DG, Stricklin WR (1981b) Bison and hereford steer digestion of sedge hay. Can $J$ Anim Sci61, 165-174

Inra (1988). Alimentation des bovins, ovins et caprins (R Jarrige, ed), Inra, Paris, $471 \mathrm{p}$

Jenning DC, Hebbring $J(1983$ ) Breeding for maximum profits. In: Buffalo Management and Marketing (National Buffalo Associations, ed), Po Box 706, Custer, États-Unis, 83-93

Kay RNB (1985) Seasonal variation of appetite in ruminants. In: Recent Advances in animal Nutrition 1985 (W Haresign, DJ Cole, eds), Butterworth, Londres, 199-210

Michalet-Doreau B, Gatel F (1988) Évolution au cours d'une année des quantités ingérées de foin par des béliers castrés. Ann Zootech 37, 151-158

Peters RR, Chapin LT, Emery RS, Tucker HA (1980) Growth and hormonal response of heifers to various photoperiods. J Anim Sci 51, 1148-1153

Petitclerc D, Chapin LT, Emery RS, Tucker HA (1983) Body growth, growth hormone, prolactin and puberty response to photoperiod and plane of nutrition in holstein heifers. J Anim Sci 57, 892-898

Richmond RJ, Hudson RJ, Christopherson RJ (1977) Comparison of forage intake and digestibility by American bison, yak and cattle. Acta Theriologica 22 , 225-230

Towne G, Nagaraja TG, Cochran RC, Harmon DL, Owensby CE, Kaufman DW (1988) Comparisons of ruminal fermentation characteristics and microbial populations in bison and cattle. Appl Environ Microbiol 54, 2510-2514

Towne G, Nagaraja TG, Cochran RC (1989) Ruminal microbia! populations and fermentation characteristics in bison and cattle fed high- and low-quality forage. Microb Ecol 17, 311-316

Thériez M (1988) Élevage et alimentation du cerf. Caractéristiques physiologiques besoins et élevage des adultes. Inra Prod Anim 1, 319-330

Thériez M (1989) Élevage et alimentation du cerf. Élevage des jeunes et production de viande. Inra Prod Anim 2, 105-116 